CAHIERS DE

NARRATOLOGIE

\section{Cahiers de Narratologie}

Analyse et théorie narratives

$28 \mid 2015$

Le récit comme acte cognitif

\title{
Vers un cinéma interactif
}

Les mutations du documentaire à l'ère informatique

Michael Lew

\section{CpenEdition}

Journals

Electronic version

URL: https://journals.openedition.org/narratologie/7259

DOI: 10.4000/narratologie.7259

ISSN: 1765-307X

Publisher

LIRCES

Electronic reference

Michael Lew, "Vers un cinéma interactif", Cahiers de Narratologie [Online], 28 | 2015, Online since 14 January 2016, connection on 18 November 2022. URL: http://journals.openedition.org/narratologie/ 7259 ; DOI: https://doi.org/10.4000/narratologie.7259

This text was automatically generated on 18 November 2022 .

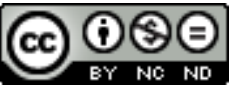

Creative Commons - Attribution-NonCommercial-NoDerivatives 4.0 International - CC BY-NC-ND 4.0 https://creativecommons.org/licenses/by-nc-nd/4.0/ 


\title{
Vers un cinéma interactif
}

\author{
Les mutations du documentaire à l'ère informatique
}

\author{
Michael Lew
}

«Do we need a new Manhattan Project?»
Eric Zimmerman
Game Design Challenge, Game Developers
Conference 2005
San Francisco

Crise des représentations

1 Le désespoir et le sentiment de vacuité sont imputables à la faillite des "grands organisateurs de récits ": récits enchanteurs et mythiques qui ont pour fonction d'aider à définir notre identité individuelle et de groupe, récits qui racontent les liens qui nous unissent ou nous opposent, qui motivent les luttes et les actions qui nous animent.

Quand éclate une manifestation, un soulèvement étudiant, ou une crise politique comme la crise des banlieues françaises de 2005, on peut se demander s'il s'agit d'une crise de la communication, d'identité, d'image, ou une rupture de confiance entre les élites productrices de sens (monde académique, maisons d'édition, groupes de médias) et le citoyen lambda doué de sens commun. Si ce dernier ne se sent pas représenté par les dirigeants ou par les médias, alors il devient nécessaire d'offrir au plus grand nombre la possibilité de communiquer et de s'exprimer de façon autopoïétique. Une telle démarche mène à la création d'un récit collectif qui permet aux puissants d'entendre le peuple, et au peuple de s'auto-organiser.

3 La doctrine de la panfictionnalité est plaisante au niveau logique, mais le citoyen d'aujourd'hui compte sur des figures d'autorité pour trancher entre le vrai et le faux, entre l'essentiel et le négligeable. Dans le cadre de la santé ou de la sécurité publique, ainsi que dans le domaine de l'éducation, il est de la responsabilité des écrivains, comme le disait Jean-Paul Sartre, mais aussi des éditeurs, d'adhérer à une éthique et à une déontologie afin d'éviter une confusion entre réalité et fiction dans l'esprit du citoyen - citoyen qui peut être confronté avant tout à des problèmes d'ordre strictement pratique (1948). 
Jean Baudrillard questionne la réalité des évènements qui nous sont relayés à travers les médias. Dans quelle mesure dois-je être impacté dans la construction de ma propre identité par les récits de guerres lointaines? Comment suis-je sensé mettre en relation mes expériences du vécu de tous les jours (ma petite histoire personnelle) avec les grands récits historiques qui se déroulent au niveau mondial?

Et si je croise dans la rue un réfugié qui vient d'Irak? Si j'entends le récit d'une prétendue victime, d'un plaignant, quel crédit accorder à ce témoin potentiel d'un délit, d'une guerre, d'une catastrophe? Jean-François Lyotard disait à ce propos que « le réel est à la charge du plaignant ».

De l'observation d'évènements à leur mise en récit, il y a un pas à franchir, comme le raconte le conte japonais Rashomôn. En effet, pour donner du sens à de l'information, pour qu'elle nous impacte, on la représente souvent sous la forme d'une histoire. Cette capacité de raconter et de comprendre des histoires, inhérente à la naissance et à l'existence du langage, jouerait, selon l'Hypothèse Narrative Forte postulée par Patrick Henry Winston du MIT, un rôle central pour l'intelligence humaine (2011).

Forme filmique à l'heure computationnelle

Depuis la fin de la deuxième guerre mondiale, l'introduction de l'ordinateur, de la cybernétique ${ }^{1}$ et des réseaux de télécommunications mondiaux ont modifié la manière dont l'espèce humaine communique. De cette période, nous avons hérité des modèles de Turing et de von Neumann comme paradigmes de l'architecture des ordinateurs, le modèle de Zuse (prototype allemand de l'ordinateur) étant resté quasiment inconnu. Dans cet article, nous voulons étudier les formes hybrides qui sont en train de naître de la collision entre ordinateur et cinéma, et que l'on désigne généralement sous l'appellation de médias interactifs.

8 Contrairement à Gene Youngblood, qui ne fait pas de distinction ontologique entre les différentes formes d'images en mouvement selon leur origine, j'estime essentiel de pouvoir distinguer entre les images produites par un geste de la main (tel que le dessin animé), par l'enregistrement automatique d'un appareil (cinéma classique), ou encore par le résultat d'une commande informatique (image de synthèse). En choisissant de s'inscrire dans la tradition cinématographique première, on se propose ici de reposer la question que posait Bazin dans Qu'est-ce que le cinéma?, et que Barthes allait explorer dans Chambre Claire, à une époque où la majorité des appareils personnels qui permettent de jouer des films ${ }^{2}$ comporte également une importante puissance de calcul encore très peu exploitée.

9 Les pratiques que nous allons décrire peuvent être regroupées sous l'appellation de cinéma interactif, tel qu'il a été inventé et défini à travers les recherches de Glorianna Davenport et de ses étudiants au MIT Media Lab dans le interactive cinema group entre 1987 et 2007, et dont l'auteur a fait partie. C'est également l'expression qu'utilise Grahame Weinbren, co-éditeur du Millenium Film Journal, dans le numéro de sa revue qu'il a dédié à la question (1995). Peter Lunenfeld a repris cette expression dans un chapitre d'une monographie sur la narratologie transmédiatique (2004), puis Bernard Perron dans un article très complet (2005). Plus récemment, Gwenola Wagon lui a dédié sa thèse de doctorat (2008).

Le interactive cinema group du MIT Media Lab

10 Le MIT Media Lab est un laboratoire de recherche et d'innovation technologique dont l'objectif principal est de développer de nouvelles formes d'expression et de 
communication avec les nouvelles technologies, dont notamment l'ordinateur. Il est né en 1985 d'un accord entre le président du MIT Jerome Wiesner et le professeur d'architecture Nicholas Negroponte, à Cambridge, Massachusetts, dans le but d'imaginer et de réaliser sous forme de prototypes des nouvelles formes de médias et d'interfaces homme-machine.

11 C'est notamment la présence de Richard Leacock (pionnier du cinéma direct ou cinéma vérité3) qui, en interaction avec les différents chercheurs en holographie (Stephen Benton), en sciences de l'éducation (Seymour Papert, disciple de Jean Piaget), en intelligence artificielle (Marvin Minsky), en visualisation (Muriel Cooper), en interfaces homme-machine (Hiroshi Ishii), et en urbanisme (William Mitchell), a permis le développement de nombreuses innovations pour le cinéma et l'informatique aujourd'hui courantes comme les logiciels de montage numériques non-linéaires et le web-documentaire. Quand Glorianna Davenport fonda son groupe de recherche sur le cinéma interactif en 1985, son projet était d'œuvrer à la création d'un «nouveau cinéma plus complexe et plus personnel, comme s'il était en conversation avec son public », en combinant la démarche de Leacock avec les nouvelles possibilités offertes par les nouvelles technologies de l'information et de la communication (TIC) en général.

12 Tout comme en narratologie Monika Fludernik est à la recherche du «naturel ", Herbert Simon, pionnier de l'intelligence artificielle, pose dans Les sciences de l'artificiel les bases d'une science consacrée à un monde de plus en plus complexe où l'interaction entre l'homme et la nature rend très ardue la distinction entre les produits de l'homme et ceux de Dieu, si l'on entend par là la Nature telle qu'elle existait avant que l'être humain ne soit capable de la modifier en profondeur, état «originel» vers lequel tendent les adeptes de l'écologie profonde tels que Arne Naess.

Les modèles informatiques du cerveau comme ceux générés par le Blue Brain project (approche analytique), ou encore les exploits de l'intelligence artificielle (approche synthétique), peuvent permettre de mieux comprendre la manière dont fonctionne l'esprit du lecteur. Notre angle d'approche est celui du champ du design et des interactions homme-machine. En effet, les œuvres que nous étudierons ici, qui sont des co-créations de l'homme et de l'ordinateur, dépendent de l'homme et de l'ordinateur pour exister, mais aussi, et surtout, du lecteur. Ces œuvres interactives peuvent être approchées comme des machines littéraires, construites par une collaboration étroite entre des ingénieurs et des auteurs.

Certes, leur intérêt réside aussi et surtout en ce qu'elles révèlent chez le lecteur, sans lequel elles n'existeraient pas. Contrairement à un livre imprimé qui donne l'impression que tout son contenu est visible sans autre forme de décodage que la lecture, l'interaction avec une œuvre interactive génère une partie, pour utiliser un terme provenant de la ludologie, et cette partie n'est qu'une trajectoire possible, actualisée, parmi un univers potentiel de virtualités ${ }^{4}$. Puisqu'il n'est généralement pas possible d'aller étudier le code source et le contenu brut d'une œuvre informatique (bien que cette méthode soit pratiquée par certains joueurs-hackeurs), tout comme il n'est pas possible d'aller sonder le cerveau d'une personne pour lire ses souvenirs, tout ce qu'a le narratologue à sa disposition est le résultat d'une interaction. Dans le cas du documentaire interactif, on peut dire qu'une séance de visionnement du film n'est qu'une promenade possible à travers un espace ou une base de données de médias préenregistrés. 

le jeu d'échecs, l'Intelligence Artificielle (IA) s'est étendue à la compréhension et à la traduction du langage naturel, la reconnaissance de formes et d'objets, l'optimisation de trajectoires, la prédiction financière. En ce qui nous concerne, nous nous intéressons à son application dans le cinéma interactif, par exemple pour diriger l'algorithme d'un moteur de montage en temps réel qui générerait des films adaptatifs au spectateur. l'IA, car il consiste à construire des machines qui peuvent apprendre : par exemple les systèmes-experts qui modélisent le savoir d'un expert et peuvent répondre à des questions savantes. Ces méthodes peuvent être utilisées pour apprendre à reconnaître les habitudes d'un utilisateur, par exemple pour synthétiser un «téléjournal » qui ne contiendrait que les rubriques qui l'intéressent.

Vers une intelligence incarnée et située

L'objectif premier du champ de l'IA, fondé par Marvin Minsky et John McCarthy, était de construire des machines artificiellement «intelligentes » ou «douées de la faculté de penser ", contrairement à la robotique qui s'intéresse avant tout à la mécanique, et donc aux mouvements et déplacements du corps et de ses membres. Rodney Brooks et Rolf Pfeifer ont redéfini le champ en s'intéressant à l'intelligence du corps, et ont démontré l'autonomie souvent sous-estimée du système nerveux périphérique. Ce mouvement de l'embodiment postule qu'il n'est pas possible de dissocier l'esprit du corps (ou encore le software du hardware). Cette approche d'une intelligence incarnée et située n'est pas sans implications sur la narratologie interactive. Car les concepteurs de récits interactifs, qui s'adaptent constamment aux nouvelles interfaces, ne nient pas le corps du lecteur. Les jeux d'intérieur (Nintendo Wii, Dance Dance Generation) ou d'extérieur (Can you see me now? de Blast Theory) impliquent littéralement le spectateur dans ses déplacements physiques chez lui ou dans la ville. De la même manière, une nouvelle narratologie doit prendre en compte le corps du lecteur-joueur.

Ainsi, l'installation Run Motherfucker run de Marnix de Nijs demande au spectateur de marcher (voire de courir) sur un tapis roulant pour progresser dans le film qui présente des vues urbaines filmées à la première personne. L'artiste a donc choisi une interface physique semblable à celle de The Legible City de Jeffrey Shaw ${ }^{5}$, mais pour les images il travaille dans la tradition de la visite interactive d'une cité existante tel que l'a réalisé plusieurs fois l'artiste américain Michael Naimark, et pour la première fois dans le Aspen Movie Map.

Le Aspen Movie Map (fig. 1) - qui peut être considéré comme un des premiers films interactifs sur ordinateur personnel- nécessitait l'emploi de deux lecteurs de vidéodisques, afin de pouvoir précharger des séquences sur un disque pendant qu'on jouait l'autre. Cette œuvre pionnière, qui est à l'origine notamment du principe de Google StreetView, permet d'explorer la ville d'Aspen sous toutes ses coutures, depuis un véhicule (telle qu'elle était à la fin des années 1970). Cette simulation d'un parcours en voiture à travers la ville, basé sur des photographies véritables, a été conçu pour donner effectivement l'impression de se déplacer dans un lieu distant « comme si on y était ». Ce sentiment de téléprésence est encore renforcé quand les actions du joueur ont un effet réel sur le monde, ce qui est le cas dans The Telegarden de Ken Goldberg; dans cette œuvre, un utilisateur peut voir et cultiver un vrai jardin à distance, à travers internet. C'est à l'artiste-chercheur Scott Fisher que l'on doit l'invention du principe de pouvoir commander un bras à distance, inauguré au Jet Propulsion Lab de la NASA, et 
préfigurant la téléopération. Equipé de data gloves, d'un casque stéréophonique et de lunettes stéréoscopiques sensibles au mouvement de la tête ${ }^{6}$, on peut manipuler des objets à distance. Jaron Lanier a qualifié cette immersion totale dans un ailleurs, immersion semblable à celle qu'on peut éprouver lors de la lecture par exemple, de réalité virtuelle.

Comme le fait remarquer Raymond Bellour dans La Querelle des Dispositifs (2012), les artistes contemporains peuvent se permettre d'explorer, à travers l'installation, les potentiels narratifs de nouvelles technologies dans des contextes tels que les musées, les galeries ou les pavillons d'expositions nationales ou universelles. C'est ainsi que la toute première projection d'un film interactif réagissant aux choix du public a été présentée à Montréal, à Expo 67, par la Tchécoslovaquie : le Kinoautomat, réalisé par Radúz Činčera (fig. 2).

21 Sa structure narrative en arborescence demande l'intervention active du public, par le biais de machines de vote disposées dans la salle : chaque spectateur peut, comme dans une assemblée législative, exprimer sa préférence en pressant un bouton rouge ou vert, quand le protagoniste du film se trouve à un moment crucial - un moment bifurcant de son histoire - où il ne peut décider seul. Chris Hales a décortiqué et fait redécouvrir cette œuvre pionnière (2005), dévoilée au public juste une année avant le printemps de Prague, et parfois considérée comme étant une satire de la démocratie.

Aujourd'hui, les bases du processus permettant au spectateur de se forger une opinion et un point de vue propres vont de pair avec la possibilité de micro-publier soi-même instantanément dans son réseau social via Facebook ou Twitter. La possibilité de rejoindre des groupes de discussion communautaires et d'y échanger des informations crée des situations communicatives à mi-chemin entre le public et le privé, entre le broadcast et le chat. En effet, les contraintes industrielles de la télédiffusion à grande échelle, qui imposaient jadis de créer un contenu de type "one-size-fits-all ", n'a plus lieu d'être à une époque où le nombre de chaînes de télévision disponibles a explosé. La stratégie des diffuseurs consiste maintenant à proposer une offre de programmes accessibles en tout temps, et on peut imaginer dans un futur proche l'apparition de contenus customisés ou "sur mesure ", disponibles en multicast, selon les préférences, choix et habitudes des téléspectateurs. Ainsi, Stefan Agamanolis, avec le projet Viper (2003), a démontré qu'il était possible de créer des films dont l'essence serait préservée mais dont la présentation serait variable (et donc allographiques, au sens de Nelson Goodman).

Le travail du lecteur-modèle

Espen Aarseth utilise le terme d'ergodicité pour définir tout texte qui demanderait du travail au lecteur afin d'être parcouru, décodé (1997). Ce travail pourrait être par exemple l'obligation, pour certains, à utiliser constamment un dictionnaire durant la lecture, de par le langage exigeant que l'auteur emploie; ou encore la nécessité de tracer des cartes, d'utiliser des atlas et encyclopédies, ou de dessiner des arbres généalogiques entiers; ou encore celle d'aller sur internet à la recherche d'informations particulières, éventuellement en collaboration avec d'autres lecteursspectateurs de la même œuvre, pour pouvoir poursuivre.

24 Cette question sur l'activité du lecteur est abordée de manière ludique par Martin Le Chevallier dans son film interactif de fiction Le Papillon, qui représente, pourrait-on dire, le degré zéro de l'interactivité (fig. 3). 
Son personnage principal (interprété par Mathieu Amalric) semble être doué pour absolument tout: il pourrait être Président de la République, artiste contemporain, curé, père de famille, ou encore voleur, anarchiste ou ermite. Quand il semble s'être fixé et stabilisé dans une étape importante de sa vie, le spectateur (qui ne peut que s'ennuyer de cette situation), est invité à cliquer, ce qui a pour effet de sortir le héros de la situation dans laquelle il se trouve et de le mettre à la recherche de nouveaux horizons. La macro-structure narrative du film est une boucle, de manière similaire au Fantôme de la Liberté de Buñuel, qui sortit en salles durant les dernières années du cinéma permanent. Si le lecteur n'agit pas, alors le personnage n'évolue pas, et reste dans une boucle sans fin.

Un autre exemple est celui de Jinxed, de Chris Hales, comédie slapstick où un jeune homme qui doit se rendre à un entretien d'embauche peut en être empêché par le spectateur. En cliquant au bon endroit de l'image (sur un hotspot), on peut, à la manière d'un malin fantôme, renverser des objets dans son appartement, provoquant des incidents qui le retarderont et lui feront rater son rendez-vous. Contrairement à la tradition du théâtre de Guignol qui permet aux enfants de coopérer avec le héros en criant aux marionnettistes la direction dans laquelle le méchant a fui, ici le lecteur peut, métaleptiquement, agir sur l'environnement matériel du héros et l'entraver, ou même le faire échouer dans sa quête.

Quelle activité doit-on demander au lecteur afin qu'il puisse continuer à lire le texte ou à voir l'œuvre? Au cinéma les images défilent toutes seules grâce au projectionniste et à son projecteur, tandis que pour la lecture individuelle il est encore nécessaire en général de pouvoir tourner les pages. Encore faut-il que le livre ait encore des pages. Ces nouveaux gestes de la lecture ont d'ailleurs fait l'objet d'un atelier à la Bibliothèque Nationale de France au printemps 2012.

L'interactivité peut permettre de tester les connaissances du lecteur, afin d'adapter l'œuvre en conséquence, par exemple pour lui apporter un éclairage sur un sujet qu'il ne connaît pas, préalable nécessaire pour exposer une thèse, ou simplement pour tester sa compréhension du matériel exposé jusque-là, afin de lui permettre de digérer et de maîtriser ce qu'il vient de lire ou de voir. Un texte ponctué de questionnaires interactifs permet à l'ordinateur de présenter à nouveau (peut-être différemment) des concepts qui n'auraient pas été bien assimilés à la première lecture ou au premier visionnement.

Pour voir un film, il suffit de rester immobile et d'avoir des yeux et des oreilles en état de fonctionnement, mais encore faut-il pouvoir cligner des yeux pour humidifier la cornée.

Dans In the Blink of an Eye, le monteur Walter Murch prétend qu'il y a un lien entre les plans de coupe et les clignements d'yeux des spectateurs, ce qui n'a pas été démontré scientifiquement. Mais la prise en compte du point de regard sur l'écran par l'œil du spectateur permet de faire des raccords convaincants ("to cut on action")

Certains spectacles vivants demandent de bouger la tête selon la configuration de la scène, mouvement qui anticipe l'invention de la caméra et de ses mouvements, et qui marque la transition du théâtre au cinéma.

Jean-Luc Godard, qui n'avait encore jamais fait de film interactif, contrairement à Alain Resnais ou Chris Marker, a exploré cette idée dans son dernier film en stéréoscopie Adieu au langage (fig. 4) : certains plans présentent à l'œil gauche et à l'œil droit deux 
points de vue complètement différents, de sorte que le spectateur est forcé de fermer l'une de ses deux paupières, ce qui lui permet de choisir entre deux caméras (A ou B), par simple clignement des yeux.

Ce principe de pouvoir changer de caméra durant le visionnement est possible sur DVD. On l'appelle communément le multi-cam. Il a aussi été utilisé à la télévision, qui, pour des raisons techniques, ne permet pas d'inclure plusieurs flux vidéo dans une seule chaîne. Une solution (peu pratique) consiste à diffuser les différents flux sur plusieurs chaînes, ce qui a été fait par la télévision danoise comme évènement du passage à l'an 2000, avec le film "D-Dag ", réalisé par les quatre fondateurs du mouvement Dogme (dont le manifeste a été déclamé par Lars von Trier au Théâtre de l'Europe-Odéon de Paris en 1995, lors de la célébration du centenaire de la première projection cinématographique). Une autre solution consiste à utiliser des boîtiers-décodeurs : la $B B C$ a ainsi diffusé des matches de tennis qui permettaient au téléspectateur depuis chez lui de choisir en direct entre plusieurs caméras. Récemment, TF1, en utilisant internet comme média de diffusion, a permis aux fans, lors de la coupe du monde de football, de choisir entre six caméras en direct. Au multi-cam s'ajoutent aussi les fonctions de replay qui permettent de revoir, ou de voir en différé, des programmes pré-enregistrés. Avec la catch-up TV ("télévision de rattrapage »), le téléspectateur s'affranchit de la temporalité imposée par les grilles de programmes, pour choisir luimême le moment de visionnement, ce qui, avant les set-top boxes à disque dur ou à disque électronique (hard disk ou solid-state) n'était possible qu'avec l'enregistrement (analogique et de mauvaise qualité) sur magnétoscope. Il peut ainsi faire des sauts dans le temps et revoir des moments-clés d'un match ou d'une émission, même au ralenti s'il le veut.

L'art cinétique n'est pas étranger à ces procédés: la sculpture d'Agam de la place Dizengoff à Tel-Aviv présente des couleurs différentes selon le point de vue du spectateur, sa position dans l'espace et ses éventuels déplacements qui modifient ce qu'il voit. Le théâtre non plus : Dans la pièce Mare's Nest de Station House Opera, le spectateur ne peut pas tout voir. En effet, selon sa position dans l'espace, il n'aura un accès visuel qu'à un côté ou l'autre de l'écran biface qui est disposé au centre d'une grande salle de type white cube. Libre de se déplacer au cours de la pièce, car non astreint à un siège numéroté, le spectateur doit toutefois choisir entre la face $\mathrm{A}$ ou la face B de l'écran central. Ce choix va déterminer ce qu'il voit, ce qu'il perçoit et ce qu'il comprend de l'action qui se déroule sous ses yeux.

En résumé, dans un film interactif, et à la différence d'un film linéaire, il survient, après ou pendant l'acte d'interprétation et de réflexion du spectateur, un acte de décision, suivi d'une action : tourner la page, cliquer sur l'écran, choisir une réponse...

Ce retour (ou feedback en anglais) du spectateur - qui par cette possibilité qui lui est offerte est devenu spectacteur - forme la base de ce qu'on pourrait appeler le processus d'auto-régulation des médias adaptatifs, tels qu'on les a déjà découverts dans la nature et décrits en cybernétique et en théorie des systèmes. Il permet à l'ordinateur de modifier le film selon l'action du spectateur.

Le mission statement du groupe de recherche de Glorianna Davenport évoque la conversation comme un idéal à atteindre entre le réalisateur et son public, car l'adaptation au locutaire est un phénomène naturel dans le déroulement d'une conversation. Les groupes de travail en dialogue inter-religieux (FEPS/FSCI, 2010) résument ce problème en disant qu'il est préférable de parler avec que de parler de. 
C'est pourquoi un grand nombre de projets de recherche récents en intelligence artificielle sont basés sur la simulation d'une discussion avec un agent intelligent. C'est le prix Loebner qui récompense annuellement la simulation la plus réaliste, par les critères du test de Turing. Alan Turing a reformulé les questions que Descartes posait sur ce qui permet de distinguer une personne vivante d'un automate (1950), non pas en termes de son apparence mais en termes de sa capacité à communiquer au cours d'une conversation.

Ainsi la vidéo-conférence, par exemple, permet de voir et d'entendre en direct une personne qui vit à un autre endroit de l'espace, mais pas du temps; seul l'enregistrement permet de restituer la présence d'une personne ayant vécu à une autre époque. Le genre du portrait interactif permettrait de simuler la conversation avec un mort ou avec un personnage de fiction. Le Portrait $n^{\circ} 1$ de Luc Courchesne figure parmi les premières réalisations de cette idée en vidéo interactive (fig. 5) : une jeune femme appelée Marie peut être interrogée par le biais d'un menu à choix multiples de questions et réponses.

A chaque sélection du spectateur, le clip correspondant est joué, contribuant à l'illusion du dialogue - en effet la conversation est pré-écrite et, contrairement au jeu Façade, ne permet pas des entrées de texte (à la voix ou au clavier) vraiment libres. A cette frustration du spectateur, Marie répond: «Il est vrai que je suis inatteignable, et que vous ne pouvez pas me changer. Mais regardez les gens autour de vous: sont-ils si différents de moi ? Sont-ils accessibles?»

Types d'interactivité

40 Notre définition de l'interactivité est basée sur la théorie de l'information de Shannon. Elle demande de pouvoir mesurer en bits la quantité d'informations qui sont échangées dans les flux entre émetteur (l'ordinateur) et récepteur (le lecteur-spectateur). On appelle interactive une œuvre dont on peut démontrer qu'elle répond ou réagit à son utilisateur selon une relation de cause à effet entre une action de l'utilisateur et une modification dans le déroulement temporel de l'œuvre.

41 Est dite interactive en surface - ou exploratoire chez Marie-Laure Ryan (2006) - une œuvre dont la présentation (syuzhet) peut être modifiée par le spectateur. Est dite interactive en profondeur - ou ontologique chez Marie-Laure Ryan (2006) - une œuvre narrative dont l'histoire (fabula) peut être modifiée par le spectateur.

42 Ainsi, Kinoautomat est une œuvre interactive en profondeur parce que les choix du public se substituent à celui du protagoniste du film: Monsieur Novak est confronté régulièrement à des situations de dilemme moral, et c'est la majorité des spectateurs qui va pouvoir prendre une décision à sa place. La structure narrative à embranchements de ce film est similaire à celle des films Smoking/No Smoking, Sliding Doors ou Lola Rennt, dont la présentation est pourtant linéaire. Le choix du spectateur peut avoir un effet de vie ou de mort sur le protagoniste.

Si l'on prend un web-documentaire récent produit par ARTE et librement disponible sur internet comme Gaza/Sderot, on constate au contraire qu'il est interactif en surface uniquement, car les choix de l'internaute ne permettent pas de modifier le rapport de forces entre Israéliens et Palestiniens dans l'œuvre; les actions du spectateur déterminent seulement l'ordre et la durée de présentation des différents clips qui montrent la vie quotidienne des habitants des deux côtés de la frontière (fig. 6). 
En revanche, le jeu vidéo PeaceMaker, qui traite aussi du conflit israélo-palestinien, est interactif en profondeur, car il permet au joueur de changer le cours de la partie selon le camp qu'il a choisi et ses décisions politiques.

La question de l'interventionnisme a été abordée par Richard Leacock en parlant de sa pratique de réalisateur de cinéma-vérité: "Nous voulions créer un genre de journalisme télé entièrement nouveau, entièrement basé sur l'observation, avec peu ou pas d'intervention ${ }^{9}$. (2011). Ayant filmé le fameux bras de fer entre JFK et le président de l'université du Mississippi dans Crisis, il montre au spectateur une situation de racisme à laquelle il faut mettre fin, mais sans prendre parti lui-même ${ }^{10}$. C'est le cas également du projet Tired of Giving In réalisé au MIT avec Carol Strohecker, qui raconte de manière interactive en surface le boycott des autobus par la communauté noire de Montgomery initié en 1955 par Rosa Parks. En effet il ne s'agissait pas pour les auteurs de permettre au spectateur de pouvoir explorer des mondes possibles contrefactuels, mais uniquement de dévoiler les évènements tels qu'ils se sont réellement déroulés à travers les voix de différents narrateurs-modèles fictifs (2001).

grand public et de la société civile aux médias, faisait la proposition suivante (1985): «Nous devons supprimer les systèmes de représentation qui portent en eux une autorité qui, dans mon esprit, est répressive, en ce qu'elle ne permet pas, ni ne fait de place aux interventions de ceux

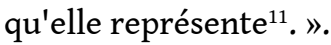

Le design d'interaction et d'interfaces

Comme nous l'avons vu plus haut, l'utilisateur d'une œuvre interactive doit faire des choix et prendre des décisions. C'est au designer d'interaction de concevoir les interfaces homme-machine ou homme-ordinateur et leur mode de fonctionnement, afin qu'elles puissent être dessinées, construites et programmées. Il ne s'agit pas seulement d'anticiper les actions de l'utilisateur et les effets de ces actions sur le déroulement de l'œuvre, mais également de proposer des interfaces intuitives. En effet, des questions comme "Que dois-je faire? », « Que peut-on faire? » se posent au lecteur confronté à ces œuvres d'un genre nouveau.

On peut faire la distinction entre les interfaces matérielles et logicielles. Manettes de jeu, joysticks, capteurs, caméras et autres périphériques d'entrée tels que la souris ou le clavier appartiennent à la première catégorie. Tandis que boutons, cases à cocher, menus, fenêtres, boîtes de dialogue et champs éditables font partie de la deuxième.

Le Kinoautomat a recours à des pupitres de vote binaire (rouge ou vert); le Aspen Movie Map à des boutons au bas d'un écran tactile (et d'une carte de repérage en haut de l'écran); Adieu au langage n'a aucune interface (sauf les lunettes 3D) et demande de fermer un œil ou l'autre quand les deux images présentent des vues non stéréoscopiques; Portrait $n^{\circ} 1$ demande de cliquer sur une des phrases qui s'affichent quand Marie s'arrête de parler.

Le tableau 1 (fig. 7) résume les différents types d'interaction utilisés pour les œuvres de cinéma interactif décrites dans cet article.

Anticipant les interfaces faisant usage du corps entier (whole body interaction) comme la Wii ou la Kinect, l'installation Office Voodoo, présentée en 2003 au Festival du Nouveau Cinéma de Montréal, permet au spectateur d'influencer les émotions des acteurs d'un sitcom expérimental qui a lieu dans un bureau (fig. 8). 

cabine de projection, et ces données sont utilisées par un moteur de montage en temps réel pour altérer le cours du film. Il s'agit donc d'une interaction en profondeur qui peut amener Frank à danser avec sa collègue Nancy ou au contraire à ignorer ses avances sexuelles. Dans un cinéma vraiment interactif, les compétences de type moteur (comme l'adresse, l'habileté, la précision, la grâce, la rapidité de réaction) courantes dans le jeu vidéo, s'ajoutent aux compétences de type cognitif (raisonnement, interprétation, prise de décision, reconnaître un bon ou un méchant) courantes dans le cinéma classique.

mode de diffusion a un impact sur le choix de l'interface. En effet, si un artiste peut construire presque ce qu'il veut pour une installation de type muséal, il n'en est pas de même pour les web-documentaires, qui doivent pouvoir être visionnés via internet sur tous les ordinateurs personnels du commerce, à l'aide d'un navigateur standard. comparaison avec les images synthétiques générées par ordinateur, mais elles ont cette qualité unique de témoigner d'un moment de réalité dans l'espace et dans le temps. Le cinéma interactif que nous avons décrit dans cet article est basé sur l'hypothèse qu'il est possible de reproduire de manière logicielle et artificielle le savoir d'un monteur, pour construire des moteurs de montage capables d'assembler instantanément et sur mesure des flux audio-visuels, en réponse à des actions du spectateur (seul ou en groupe). Pour satisfaire ce dernier dans son horizon d'attente, Csiksentmihalyi a proposé le concept de flow repris par le concepteur de jeux Jenova Chen (2007), qui décrit en psychologie l'état dans lequel se trouve un individu qui a du plaisir à poursuivre une activité, en lui évitant l'ennui ou l'anxiété, et en lui opposant une résistance à la mesure de ses capacités. En effet, un système ou une œuvre trop difficiles vont décourager les plus faibles, qui n'en arriveront pas au bout et seront tentés d'abandonner ou de trouver des raccourcis. 
La théorie du flow suggère donc de créer des médias adaptatifs, qui résulteraient de la nature programmable des supports computationnels disponibles aujourd'hui pour lire ou voir des films. Ces nouveaux types de récits interactifs encouragent le spectateur à intervenir, à faire entendre sa voix, et à découvrir l'effet qu'il peut avoir sur le monde à travers des simulations de la réalité. Particulièrement adaptées à l'exploration des mondes possibles, ces œuvres ont aussi l'avantage de permettre aux auteurs de déléguer une partie de leur pouvoir décisionnel au destinataire du récit, et de le faire participer pleinement à l'acte de création.

Mes remerciements à Glorianna Davenport, Scott

Fisher et Jeffrey Huang pour leur soutien dans mon travail de recherche, ainsi qu'à Victoria Martin, Ilan Lew et Guenda Bernegger pour leurs précieux retours et commentaires.

\section{BIBLIOGRAPHY}

Aarseth, Espen. Cybertext : perspectives on ergodic literature, Baltimore \& London: Johns Hopkins University Press (1997).

Agamanolis, Stefan, and V. Michael Bove Jr. Viper: A framework for responsive television, IEEE Multimedia 10.3 (2003) : 88-98.

Bellour, Raymond. La Querelle des dispositifs, P.O.L. (2012).

Chen, Jenova. Flow in games (and everything else), Communications of the ACM 50.4 (2007) : 31-34.

Commission de dialogue entre juifs et protestants. « Ensemble sur le chemin du respect :

Déclaration commune sur le dialogue entre juifs et protestants en Suisse », FEPS / FSCI, (2010).

Crawford, Chris. «The Art of Computer Game Design », (1982), disponible en ligne :

http://www-rohan.sdsu.edu/ stewart/cs583/

ACGD_ArtComputerGameDesign_ChrisCrawford_1982.pdf

Davenport, Glorianna, and Murtaugh, Michael. ConText: towards the evolving documentary, ACM Multimedia, (1995): 377-378.

Hales, Chris. Cinematic interaction: from kinoautomat to cause and effect, (2005): 54-64. vol. $16, \mathrm{n}^{\circ} 1$. Leacock, Richard. The Feeling of Being There, a Filmmaker's Memoir, Semeïon Editions (2011).

Lévy, Pierre. Cyberculture, Rapport au Conseil de l'Europe, Editions Odile Jacob (1997). 
Lunenfeld, Peter. « The myths of interactive cinema, » in M.-L. Ryan (ed.) Narrative across Media: The Languages of Storytelling. University of Nebraska press (2004): 377.

Morin, Edgar. « Pour un nouveau cinéma-vérité ». France-Observateur, n 506, 14 janvier 1960.

Perron, Bernard. « Le cinéma interactif à portée de main. » The Five Senses of Cinema, XI International Film Studies Conference, Udine, Forum (2005).

Ryan, Marie-Laure. Avatars of story. University of Minnesota Press (2006).

Said, Edward. « In the Shadow of the West », Wedge 7-8 (1985): 4-11.

Sartre, Jean-Paul. « La responsabilité de l'écrivain. » (1946)

Strohecker, Carol, et al. « Tired of Giving In: An Experiment in Narrative Unfolding ». in Narrative Intelligence: Advances in Consciousness Research, Amsterdam, Philadelphia: John Benjamins, (2001).

Turing, Alan M. Computing machinery and intelligence, Mind (1950): 433-460.

Wagon, Gwenola. « Utopies d'un cinéma interactif. » PhD diss., Atelier national de reproduction des thèses, Suisse (2008).

Weinbren, Graham. « In the Ocean of Streams of Story », in Interactivities, Millennium Film Journal, no. 28, (1995).

Winston, P. H. « The Strong Story Hypothesis and the Directed Perception Hypothesis », in AAAI Fall Symposium: Advances in Cognitive Systems (2011).

\section{APPENDIXES}

\section{Table des Illustrations}

Fig. 1. Aspen Movie Map

Fig. 2. Kinoautomat

Fig. 3. Le Papillon

Fig. 4. Adieu au langage

Fig. 5. Portrait $n^{\circ} 1$

Fig. 6. Gaza / Sderot

Fig. 7. Tableau 1 : Types d'interactivité dans le film interactif

Fig. 8. Office Voodoo

Fig. 9. JBW Portrait 
Fig. 10. Tableau 2 : Evolution des technologies pour le film interactif

\section{Corpus}

Chris Hales, Jinxed, Royaume-Uni, 1998

Martin Le Chevallier, Le Papillon, France, 2005

Michael Lew, Office Voodoo, Suisse, 2002

Raduz Cincera, Kinoautomat, Tchécoslovaquie, 1967

Luc Courchesne, Portrait ${ }^{\circ} 1$, Canada, 1990

Jean-Luc Godard, Adieu au Langage, Suisse, 2014

Michael Murtaugh, JBW portrait, USA, 1995

Susanna Lotz, Joël Ronez, Alex Szalat, Gaza/Sderot, ARTE, Allemagne, 2009

Ian Flitman, Hackney Girl, Royaume-Uni, 2002

Architecture Machine Group, Aspen Movie Map, USA, 1978

\section{NOTES}

1. Cette théorie, qui a beaucoup d'applications chez les ingénieurs en automatique, est souvent résumée par le fonctionnement d'un thermostat: si la température est trop élevée, alors le système va enclencher la ventilation; si la température est trop basse, il va allumer le chauffage. Cette théorie révolutionnaire apparue après la deuxième guerre mondiale se veut caractéristique $\mathrm{du}$ fonctionnement adapté d'un système, inspirée des découvertes de la régulation biologique chez les êtres vivants telle que l'homéostasie. La cybernétique est une théorie de la communication et de la circulation des signaux chez l'homme et l'animal proposée par Norbert Wiener en 1948.

2. L'utilisation du terme « film » n'implique plus que les images aient été enregistrées sur un film d'halogénures d'argent (soit de la pellicule): il inclut également la vidéo analogique et numérique (DV SD et HD).

3. C'est à Edgar Morin que l'on doit la reprise (ou la réinvention), en 1960, de l'expression de « cinéma vérité » de Dziga Vertov.

4. De la même manière dont Saussure disait que lorsqu'on étudie une langue, tout ce qu'on a ce sont des paroles (c'est-à-dire des manifestations spontanées d'un système linguistique sousjacent), lorsqu'on joue à un jeu vidéo ou à une œuvre interactive, on a des "parties ", des trajectoires dans le monde des possibles définis par le moteur de jeu. Il convient alors de reconstituer le jeu dans son ensemble et dans ses principes, en étant conscient qu'on a peut-être manqué des choses importantes. Comme le dit un proverbe irlandais : «I know Dublin even though I haven't seen all of its streets ".

5. Cette installation de Jeffrey Shaw demande au spectateur de pédaler sur un vélo pour explorer une ville dont les immeubles sont d'immenses lettres de l'alphabet générées en temps réel.

6. Il s'agit du BOOM (Binocular Omni Orientation Monitor), ancêtre de l'Oculus Rift actuel.

7. Cf. le blog du Labo de la BNF et l'article «Etude sur le livre numérique et les français en 2014 » (lien vérifié le 23 juillet 2015).

8. C'est le psychologue russe Alfred Yarbus qui en premier a étudié les mouvements oculaires lors de l'observation d'une image; l'oculométrie permet aujourd'hui d'enregistrer précisément ces derniers afin d'adapter la mise en page d'un texte ou le ciblage d'une publicité. 
9. "We wanted to create an entirely new genre of TV journalism based on observation with little or no intervention" (traduction de la rédaction).

10. De la même manière que beaucoup d'écoliers et d'étudiants juifs se sont vu interdire l'accès aux institutions publiques d'enseignement en Europe quant ont été édictées les lois raciales autour de 1935-1937, l'université du Mississippi n'acceptait pas d'étudiants noirs avant 1962, et ce indépendamment de leurs capacités intellectuelles ou financières, de leurs aspirations professionnelles ou même de leurs résultats scolaires.

Cet épisode de la déségrégation de Ole Miss a été filmé par Richard Leacock et constitue sa contribution au film Crisis. Ce documentaire met en scène le bras de fer entre JFK et le recteur de l'université, pour permettre au premier étudiant noir d'entrer à l'université. Le combat du président américain contre l'apartheid qui régnait au Sud des Etats-Unis fut un signal fort en faveur de la méritocratie et de la diversité, contre la reproduction des élites.

11. "What we must eliminate are systems of representation that carry with them the authority which, to my mind, has been repressive because it doesn't permit or make room for interventions on the part of those represented." (traduction de la rédaction)

\section{ABSTRACTS}

Inaugurated with the Kinoautomat in 1967, interactive cinema is finally accessible to everyone. This article examines the particularities of these films who are played in a non-linear fashion and are adaptive to the viewers. By taking into account instantaneously the decisions of the audience, are they an accomplishment of the cybernetic promise?

Through an illustrated case study, we introduce some useful notions in order to extend the field of contemporary narratology to the study of these new artworks.

Michael Lew est un artiste-chercheur indépendant né en 1977. Après avoir obtenu son Master ès Sciences en ingénierie électronique à l'EPFL sur le thème de l'intelligence artificielle, il a été Research Fellow au Media Lab Europe, laboratoire européen du MIT, puis Adjunct Professor et Visiting Scholar à USC dans le département de médias interactifs. En 2012, il a bénéficié d'un soutien du Fonds National Suisse de la Recherche Scientifique dans le cadre du projet sinlab.

Apparu en 1967 avec le Kinoautomat, le cinéma interactif devient, avec les web-documentaires, enfin accessible à tous. Cet article examine les particularités de ces films dont le déroulement est non-linéaire et adaptatif aux spectateurs. La prise en compte instantanée des décisions du public permet-elle de réaliser la promesse cybernétique?

A travers une étude illustrée de cas, nous proposons des notions utiles pour étendre le champ de la narratologie contemporaine à l'étude de ces nouveaux objets d'art. 
INDEX

Keywords: aesthetics, art history, semiology, language sciences, communication sciences, interactive cinema, interactive storytelling, mass media

Geographical index: Europe, Etats-Unis, Canada

Mots-clés: esthétique, histoire de l'art, sémiologie, sciences du langage, sciences de la communication, cinéma interactif, récit interactif, médias de masse

Chronological index: $1967-2014$ 